\title{
Vent control as a means of enhancing airbag performance ${ }^{1}$
}

\author{
Richard E. Zimmermann \\ Zerad, Inc., 425 East Greenway Drive, Tempe, AZ 85282-6938, USA
}

Received 24 October 2000

\begin{abstract}
Typical automotive airbag systems have a fixed area vent for exiting gasses. The US Army Cockpit Airbag System (CABS) is unvented to prolong the period during which the system can provide occupant protection during extended helicopter crash scenarios. In each application, system performance may be enhanced by providing a controlled vent area. This paper describes work conducted under a Phase I SBIR program sponsored by the NASA Langley Research Center. The work was focused on eventual inflatable restraint system applications in general aviation aircraft, and showed that appropriate vent control offers many enhancements. Two series of tests conducted during Phase I showed that inflatable restraint system size and weight can be reduced without degrading performance, injury potential in an out of position situation (OOPS) deployment can be reduced, and peak bag pressures can be reduced (at any temperature) during normal operation.
\end{abstract}

\section{Introduction}

The primary project objective was to make occupant protection airbags more efficient by controlling the flow of vented gas. Another objective was to reduce the probability and severity of airbag induced injury in situations where the occupant is initially too close to the deploying airbag. The immediate application of interest was general aviation aircraft, but application to other airbag systems are obvious. Project tasks included valve concept development, preliminary analysis of system performance with the valves, and two series of tests evaluating the performance of a prototype valve assembly.

The valve design must provide rapid initial opening upon occupant contact with the bag as well as appropriate follow on area variation. Other factors considered in a design tradeoff study included the following:

- Functionality: Will a valve using the concept be able to control the flow as required?

- Adaptability: Assuming the valve concept can be used to control the flow, can a product applying the

\footnotetext{
${ }^{1}$ Presented at the USAARL Inflatable Restraints in Aviation Workshop, Huntsville, AL.
}

concept be included in a practical airbag module given reasonable weight and volume constraints?

- Durability: Will the components of any product based on the concept remain functional for the life of an airbag module?

- Cost: Can needed components be produced for a cost that will permit their inclusion in an airbag module?

- Ease of Assembly: Can a valve based on the concept be economically installed (OEM and service) in an airbag module?

Various valve concepts were developed, and a tradeoff study was conducted to identify the one must suitable for the airbag application. A detailed design of the preferred configuration was prepared, and a prototype valve assembly was fabricated. The prototype valve provided six 1.25 inch diameter ports, and selectable relief pressures of 3, 4 and 5 psi. The details of the valve design are not disclosed in this paper because the patent application is still in process.

\section{Analysis}

Simplified and expedient numerical analyses used in Phase I applied fundamental engineering principles to 
predict pressure, displacement, and force as a function of time. Use of the simplified analysis in Phase I provided adequate results for supporting preliminary design work, and also conserved Phase I resources, permitting more testing. MATLAB was used for this simplified numerical analysis. More sophisticated analytical tools, such as MSC.DYTRAN, will be used in follow on development to optimize the prototype system designs and predict injury criteria before testing.

The analytical steps used in the analysis were as follows:

- Using initial bag pressure and volume, occupant deceleration was integrated to obtain velocity and displacement.

- The increment of displacement was used to calculate a new bag pressure due to compression.

- Orifice flow equations were used to calculate an increment of mass flow rate, given the vent valve design characteristics.

- The reduction in bag pressure due to the increment of exiting gas flow was calculated.

- The resulting bag volume and gas characteristics were used to calculate a new increment of force on the occupant.

- Incremental calculations for fractions of a millisecond were computed for the total bag compression.

Once the MATLAB model was verified against existing pallet airbag test data it, was used to model the performance of the vent valve concept and assist in the development of the prototype valve.

\section{Test procedure}

The test procedures were designed to demonstrate the feasibility of using an exhaust vent valve to improve the performance of occupant protection airbags. There were two series of tests: ride-down tests and OOPS tests. The ridedown tests evaluated the performance of the airbag as it performs its normal function of decelerating the occupant's mass during a crash. The OOPS tests evaluated changes in potential airbag induced injury during an out of position situation (occupant too close to airbag during deployment).

\subsection{Ride-down tests}

Test method: The general test method is illustrated in Fig. 1. A custom made airbag was attached to a housing

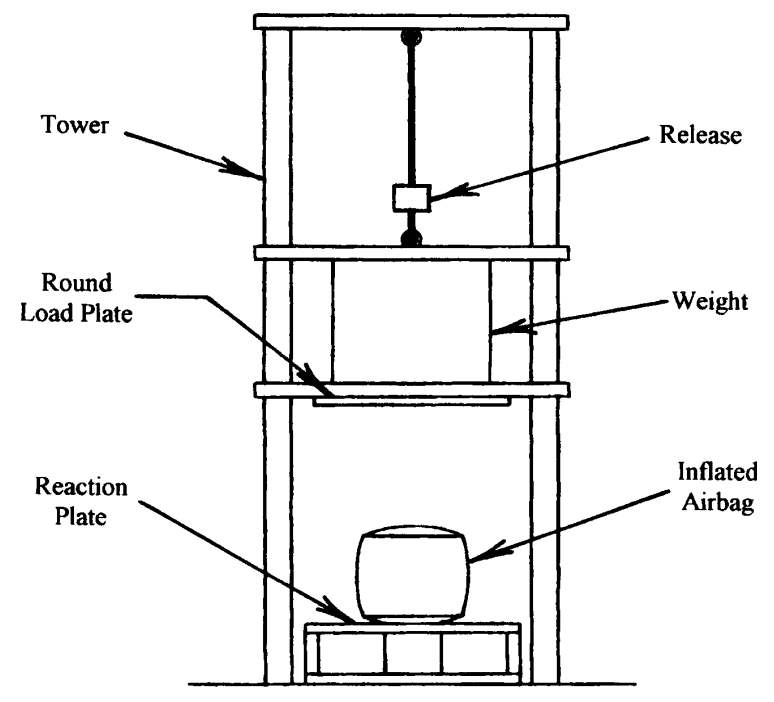

Fig. 1. Ride-down test setup.

in which the vent valves were installed. On the other end of the housing, a plate sealed the inflator opening. The housing was installed in a fixture beneath the drop tower, where a weight released from preplanned heights could compress the bag. The airbag was preinflated with compressed air to simulate inflation, and the falling weight compressed the bag to simulate occupant impact. The air bag used in the ridedown tests was cylindrical to approximate the performance predicted by the analysis. Both the diameter and the height of the bag was 16 inches. The deceleration of the weight and the pressure in the bag were measured throughout the event and recorded. Tests were also conducted without vent valves to provide a baseline (representing "as is" auto airbag designs).

Test matrix: Tests with each of the three vent valve pre-loads (3, 4, and 5 psi) and baseline tests were conducted for nine different conditions. Weights of 30,60, and 100 pounds were used, and initial velocities were 10,15 , and $22 \mathrm{ft} / \mathrm{sec}$. Free fall drop heights corresponding to the three initial velocities were $1.55,3.49$, and 7.50 feet. A total of 36 different tests were performed.

Weights: The weights used in the tests simulated the effective weight of the occupant wearing lap belt and single shoulder harness. This effective weight that will load an airbag was estimated at $1 / 3$ of total body weight. This estimate was based on experience and literature showing that an occupant wearing a three-point restraint applies approximately $2 / 3$ of the inertial load to the lap belt restraint. While appropriate for general aviation applications where the occupants will be wearing primary restraints, the same assumptions have not 
been applied in the design of airbags for automobiles manufactured in the United States. Unlike auto airbags manufactured in Europe, those airbags are required to provide protection even if a primary restraint is not worn [1].

Instrumentation: Two pressure sensors and two accelerometers were used in the ride-down tests. Both accelerometers were mounted on the dropped weight, and both pressure sensors were installed in ports in the valve housing. A small infrared lamp and photosensor were used for a trigger. A small metal flag attached to the fixture with the drop weight passed between the lamp and sensor. The resulting voltage change triggered the data acquisition system. The trigger was positioned on the tower to generate the triggering voltage just before the weight made contact with the airbag.

For the baseline tests, four of the vent valves were clamped tight shut, and air was allowed to exit from two completely open ports. A trip wire was set to release dump valves over the two "open" ports as the falling weight touched the top of the bag. These valves over the "open" ports were used to conserve compressed air. The proper initial pressure could not be achieved in the bag without them. This arrangement successfully simulated a bag prefilled with air as it was vented by a pair of (two) 1.25 in. diameter holes.

For the baseline tests, the vent hole size and number were chosen as typical of existing automotive airbags. For some time, airbags had a vent hole approximately 2 in. in diameter. Some newer bags, apparently of the "depowered" type, have a pair of vents that are each 1 in. in diameter. Therefore, vent area in US auto airbags ranges from 1.5 to 3.1 square inches, and the test setup used a vent area of 2.5 square inches. Page 9 of reference 2 provides information on typical vent areas used in earlier airbag designs.

\subsection{OOPS tests}

The OOPS tests were conducted by deploying airbags with a barrier positioned to prevent complete bag inflation.

Test method: A gas generator was installed in the prototype valve housing which was in turn installed in a test fixture (see Fig. 2). An airbag was attached to the valve housing, and an adjustable barrier attached to the fixture prevented full deployment of the airbag. The folded airbag was deployed, and bag pressure was measured. This process was repeated for a baseline condition without vent valves. The reduction in peak pressure achieved with the vent valve configuration is an

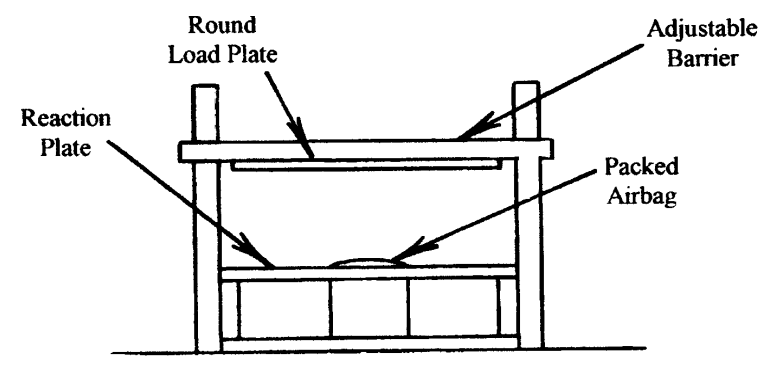

Fig. 2. OOPS test fixture.

approximate indication of reduced probability and/or reduced severity of airbag induced injury. The tests were repeated with the barrier positioned at three different distances from the undeployed airbag. The fixture was the same as that used in the ridedown test, but vertical supports were added to mount the barrier.

The airbags used in this test series were round (28inch diameter) 60-liter airbags similar to drivers' side airbags used in US automobiles. Tethers were not installed because they would have served no purpose with a barrier obstructing full bag deployment. The bags were folded in a typical automotive fold pattern, and tape was used to approximate the opening force of the plastic enclosure included in production configurations. The bag fabric was 420-denier Nylon 66, plain woven with neoprene coating on one side.

To perform the baseline tests, all vent valves were removed. Four ports were blocked, and the other two ports remained open to simulate the vents in conventional airbags.

The gas generator was ignited by manually closing a switch connected to a 12 VDC auto battery. The inflator was a new non-azide model D 60 automotive inflator provided by Talley Defense Systems of Mesa, Arizona. The inflator is being marketed by a Talley subsidiary called AEGIS Technologies, and is much smaller and lighter than the earlier azide type inflators. The smaller size makes it much more attractive for potential use in a general aviation application.

Test matrix: Six different types of OOPS tests were conducted. The tests included distances of 4, 8, and 12 in., and were performed with two different valve configurations (vent valve and baseline). The vent valve tests were conducted with the 5 psi pre-load configuration, and the baseline tests were conducted with four ports blocked and two ports open. Each of the six tests was conducted twice to demonstrate repeatability. In addition, two tests were run with the 3 psi pre-load vent valve configuration. 
Table 1

Strokes for 100 pound weight (inches)

\begin{tabular}{ccccc}
\hline Height & \multicolumn{4}{c}{ Valve } \\
\cline { 2 - 5 }$(1 / 100 \mathrm{ft})$ & $3 \mathrm{psi}$ & $4 \mathrm{psi}$ & $5 \mathrm{psi}$ & baseline \\
\hline 750 & 12 & 11.7 & 11.2 & 14.3 \\
349 & 8.5 & 8 & 8 & 11.4 \\
155 & 6.6 & 6.4 & 6.2 & 10.2 \\
\hline
\end{tabular}

Instrumentation: Two identical pressure sensors were installed in the housing between the airbag and the gas generator.

\section{Test results}

\subsection{Results of ridedown (drop) tests}

Data in the ridedown tests was collected at 10,000 samples per second and then filtered (post test) with an SAE J211 Class 60 digital filter. The filter software was an MS-Excel implementation of the SAE filter that was provided by The FAA Civil Aeromedical Institute (CAMI). Then, the two pressure and acceleration channels were averaged. Finally, the averaged acceleration was integrated twice to obtain velocity and displacement. The data was averaged, integrated, and plotted with MS Excel 97.

The tables summarize strokes required to bring the velocity of the falling weight to zero. There is a separate table for the tests with the 100,60 and 30 pound weight. Each of those tables contains information for the various valve configurations and impact velocities.

Summary of stroking distances in ridedown tests: Tables 1-3 summarize the average stroke for each valve type and drop height. These results demonstrate feasibility of the concept, but not the ultimate capability of the valve design. No attempt was made to optimize performance in Phase I.

Performance was improved the most with the 60 pound weight. Appropriate adjustment could presumable improve performance for other weights.

\subsection{Results of OOPS tests}

Table 4 shows the average peak pressures obtained in the various tests.

Table 5 shows the time the airbag pressure was over 6 psi.

The last columns in the above two tables show the percentage difference between the baseline condition (two 1.25 in. open ports) and the vent valve with the 5 psi relief pressure.
Table 2

Strokes for 60 pound weight (inches)

\begin{tabular}{ccccc}
\hline Height & \multicolumn{4}{c}{ Valve } \\
\cline { 2 - 5 }$(1 / 100 \mathrm{ft})$ & $3 \mathrm{psi}$ & $4 \mathrm{psi}$ & $5 \mathrm{psi}$ & baseline \\
\hline 750 & 12 & 11.5 & 8 & 10 \\
349 & 10 & 9.5 & 6 & 9 \\
155 & 9.9 & 8.6 & 5 & 8 \\
\hline
\end{tabular}

Table 3

Strokes for 30 pound weight (inches)

\begin{tabular}{ccccc}
\hline Height & \multicolumn{4}{c}{ Valve } \\
\cline { 2 - 5 }$(1 / 100 \mathrm{ft})$ & $3 \mathrm{psi}$ & $4 \mathrm{psi}$ & $5 \mathrm{psi}$ & baseline \\
\hline 750 & 10 & 10 & 10 & 10 \\
349 & 8.2 & 7.8 & 7.5 & 8.7 \\
155 & 7.5 & 7.6 & 7.2 & 7.2 \\
\hline
\end{tabular}

Table 4

Average peak pressures for each valve configuration (psi)

\begin{tabular}{ccccc}
\hline \multirow{2}{*}{$\begin{array}{c}\text { Distance } \\
\text { (in.) }\end{array}$} & \multicolumn{3}{c}{ Valve } & $\%$ base/5 psi \\
\cline { 2 - 4 } & $3 \mathrm{psi}$ & $5 \mathrm{psi}$ & baseline & \\
\hline 12 & N/A & 9 & 14.8 & 164 \\
8 & 8.5 & 7.3 & 11.4 & 156 \\
4 & N/A & 8.6 & 19.5 & 226 \\
\hline
\end{tabular}

\section{Discussion}

The tests demonstrated that the vent valve can control the flow of exiting gas and shorten the required stroke in a number of ways.

First, the pre-load on the valve can establish the desired pre-charge in the bag at the time of occupant impact. Creating as high a pre-charge pressure as practical is the most effective way to reduce total stroke requirements, because additional deceleration is provided at the time the velocity is highest. However, the benefits of increased initial pre-charge must be traded off against the requirement to limit the potential for injury in an OOPS.

Secondly, the characteristics of the opening (and open) valve can influence the peak acceleration reached during occupant ridedown. A valve with a lower spring constant will open faster, allow gas to escape more quickly, and reduce the peak acceleration. The maximum area of the valve in the full open position also affects the rate of initial gas flow. However, higher peak pressures which limit total stroke must be traded off against injury criteria such as the HIC.

Finally, the valve spring rate can also influence the remaining stroking distance required following peak pressure. If the valve closes faster following peak bag pressure, it keeps bag pressure higher during the final portion of the stroke. This keeps the final acceleration 
Table 5

Average time over 6 psi - msec

\begin{tabular}{ccccc}
\hline Distance & \multicolumn{3}{c}{ Valve } & $\%$ base/5 psi \\
\cline { 2 - 4 } (in.) & $3 \mathrm{psi}$ & $5 \mathrm{psi}$ & baseline & \\
\hline 12 & N/A & $4.5 \mathrm{msec}$ & $6(\mathrm{msec})$ & 133 \\
8 & $4 \mathrm{msec}$ & $3.5 \mathrm{msec}$ & $7.5(\mathrm{msec})$ & 214 \\
4 & N/A & $8 \mathrm{msec}$ & N/A & N/A \\
\hline
\end{tabular}

higher, and also contributes to reducing the total stroke requirement.

The cold air ridedown test data does not represent a one hundred percent accurate quantitative representation of how the airbag will perform when inflated with a hot gas. Being less dense due to temperature, the gas will have a different effective orifice or valve coefficient. Compensation for this effect will be included in the final valve design. Experience with cold air testing of preliminary airbag designs assures that the tests conducted thus far do demonstrate the feasibility of the air bag vent valve concept whether used with either hot or cold gas.

The benefit of the vent valve in an OOPS may actually be many times greater than shown in the preliminary feasibility tests in Phase I. Smaller baseline vents, as now used in depowered airbags, would likely have shown even greater benefits. The total vent area in the Phase I baseline tests was nearer the high end of the vent area ranges for auto airbags, and the tests still demonstrated an obvious benefit. Also, the Talley D 60 inflator used in the Phase I tests is a new generation automotive airbag inflator. If the tests had been conducted with a more aggressive inflator of an earlier design, the OOPS tests would probably have shown a greater benefit associated with the prototype vent valves.

Furthermore, existing auto airbag vents may not even be exposed in some OOPS. The OOPS tests were conducted with two open 1.25-in. diameter ports. In typical automotive airbag modules, the vents are in the bag, not in the housing. Therefore, the bag must unfold before any gas flow can reach the vents. In a very close OOPS, the effective vent area may be essentially zero rather than the baseline vent area used in the tests. In such cases, the "as is" airbag configuration would be far more lethal in an OOPS than was indicated in the tests. Therefore, the data is indicative of valve performance, but more testing is required to demonstrate the total benefit of the system relative to typical automotive airbag systems.

Finally, the baseline condition plots are valid only for bags with exactly the same pre-charge. In an actual airbag application with orifice vents only, the precharge is not necessarily closely controlled. For ex- ample, temperature extremes greatly change the performance of hot gas inflators, and can therefore cause significant changes in the pre-charge.

Development to this point has made it apparent that the vent valve concept has applications other than the intended general aviation and automotive markets. For example, the US Army cockpit airbag system (CABS) for helicopters may benefit from inclusion of such a valve. These airbag systems are not vented like auto airbags are. The reason is that the typical crash scenario is much more protracted (ex: tree strikes prior to ground impact), and longer period of bag inflation is required. The unvented approach provides enhanced protection even with a hot gas generator, which fills the bag with hot gases that rapidly contract due to cooling. However, the design and production of the inflator must be very precise to achieve the proper initial pressure. This is particularly difficult to achieve under the temperature extremes under which these helicopters operate. Inclusion of the NASA sponsored vent valve concept in the CABS system may be beneficial. Exactly the right pressure could be provided at bag inflation under all temperature conditions, and the pre-loaded valve would seal the bag (as desired) after inflation to the proper pressure. Inclusion of the valve would also greatly reduce rebound when the occupant did load the airbag. This rebound, quite energetic with a sealed bag, was initially shown to not present an unacceptable injury risk for the Army aviator population wearing protective equipment. However, elimination of this rebound may provide a highly desirable system enhancement.

\section{Conclusions}

The Phase I tests have demonstrated the feasibility of the basic concept for air bag vent control. In addition, the prototype valves designed and tested were shown to have many advantages over other valve configurations. An additional advantage of the vent valve is that it introduces a practical way to adjust valve performance for occupant size. The feasibility of this adjustment feature was demonstrated in the tests as well as the original objectives of reducing airbag size and weight and reducing lethality in an OOPS.

Specific conclusions are as follows:

- The response time of the vent valve design is fast enough for the intended function of regulating air bag exhaust flow. Tests at occupant/bag impact 
velocities of up to $22 \mathrm{ft} / \mathrm{sec}$ showed satisfactory performance. Component tests also showed that valve response time is negligible relative to system requirements.

- The vent valve can improve air bag efficiency as proposed.

- The valve may be easily adjusted to optimize performance for different occupant sizes.

- Some further development is required to achieve optimum adjustability over the entire range of performance conditions.

The OOPS tests showed that the vent valve design can also alleviate injuries associated with an OOPS. The tests showed that even the vent valves with the highest pre-load produced less peak pressure than did the baseline vent configuration. The tests with the vent valves also had significantly less pressure persistence time over 6 psi. Since injury criteria are highly dependent upon the duration as well as the peak value of contact forces, the second finding is especially significant.

The vent valve may have applications in other than the intended general aviation and automotive markets. The US Army cockpit airbag system (CABS) can probably benefit from inclusion of the vent valve. The valve would assure proper pressurization of the unvented bag configuration under all temperature extremes, and would minimize undesired rebound. Airbag systems other than those used for occupant protection might also benefit from inclusion of such a valve. Planetary landing systems and aircraft escape capsules (such as previously used on the F-111) may be other potential applications.

\section{Acknowledgements}

Funding for this development work was provided by the NASA Langley Research Center. Lisa Jones was the technical monitor.

Prior work on airbag vent valves was conducted by the US Army Natick Research, Development, and Engineering Center [3] and by Warrcik \& Associates of Prescott, Arizona. The application involved aerial cargo delivery.

\section{References}

[1] D.J. Romeo, Mid Atlantic Driver Air Bag, paper presented at the 14th International Technical Conference for Enhanced Safety of Vehicles (ESV), Muncih, Germany, May 23-26, 1994, Paper number 94-54-0-10.

[2] W. Reidelbach and H. Scholz, Advanced Restraint System Concepts, SAE Congress and Exposition, Cobo Hall, Detroit, February 26-March 2, 1979, SAE paper number 790321.

[3] N. Rosato, Design Development of an Airbag Vent control Mechanism, presented at the ASME Winter Annual Meeting, Atlanta, GA, Dec 1-6, 1991, ASME paper 91-WA-DE-1. 

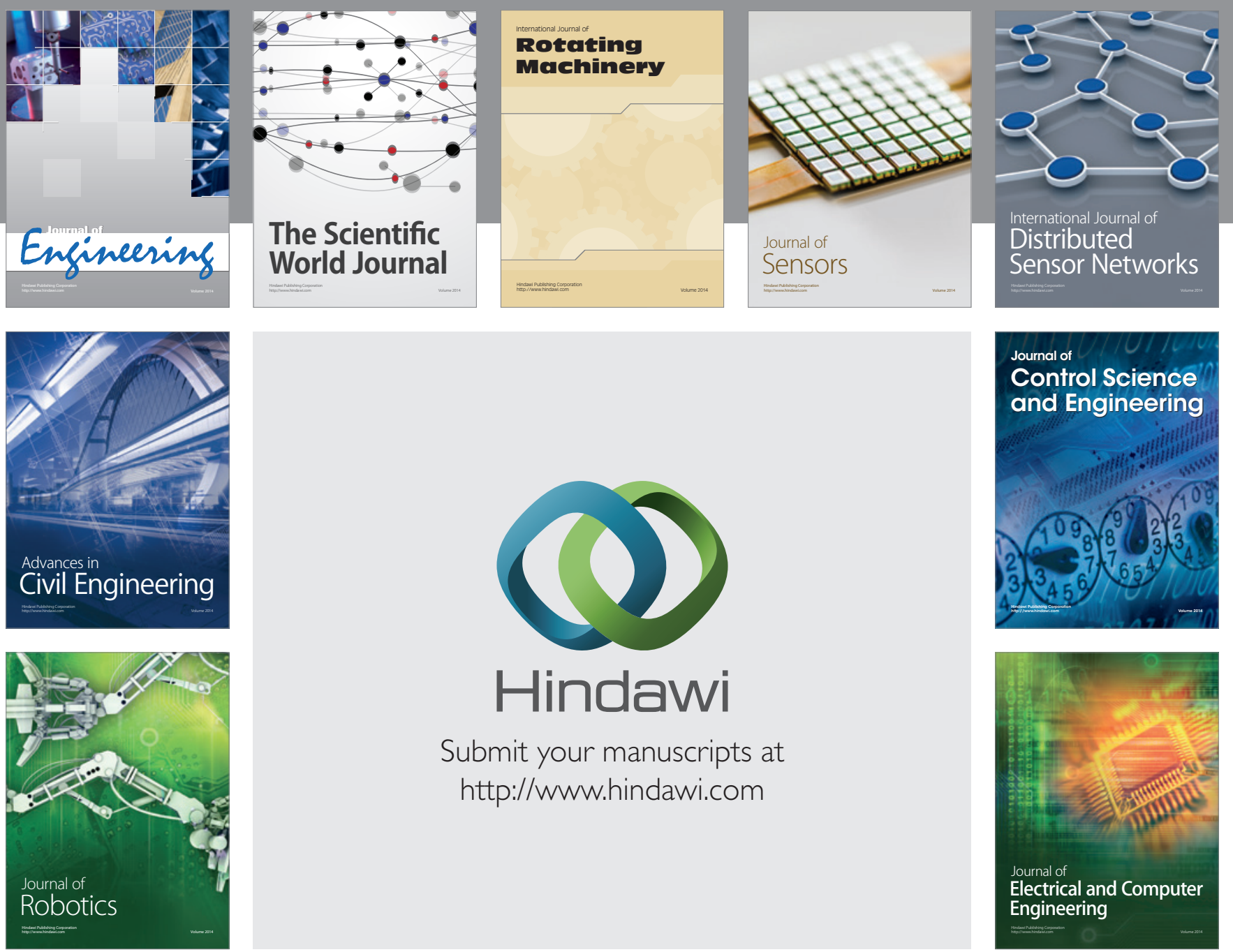

Submit your manuscripts at

http://www.hindawi.com
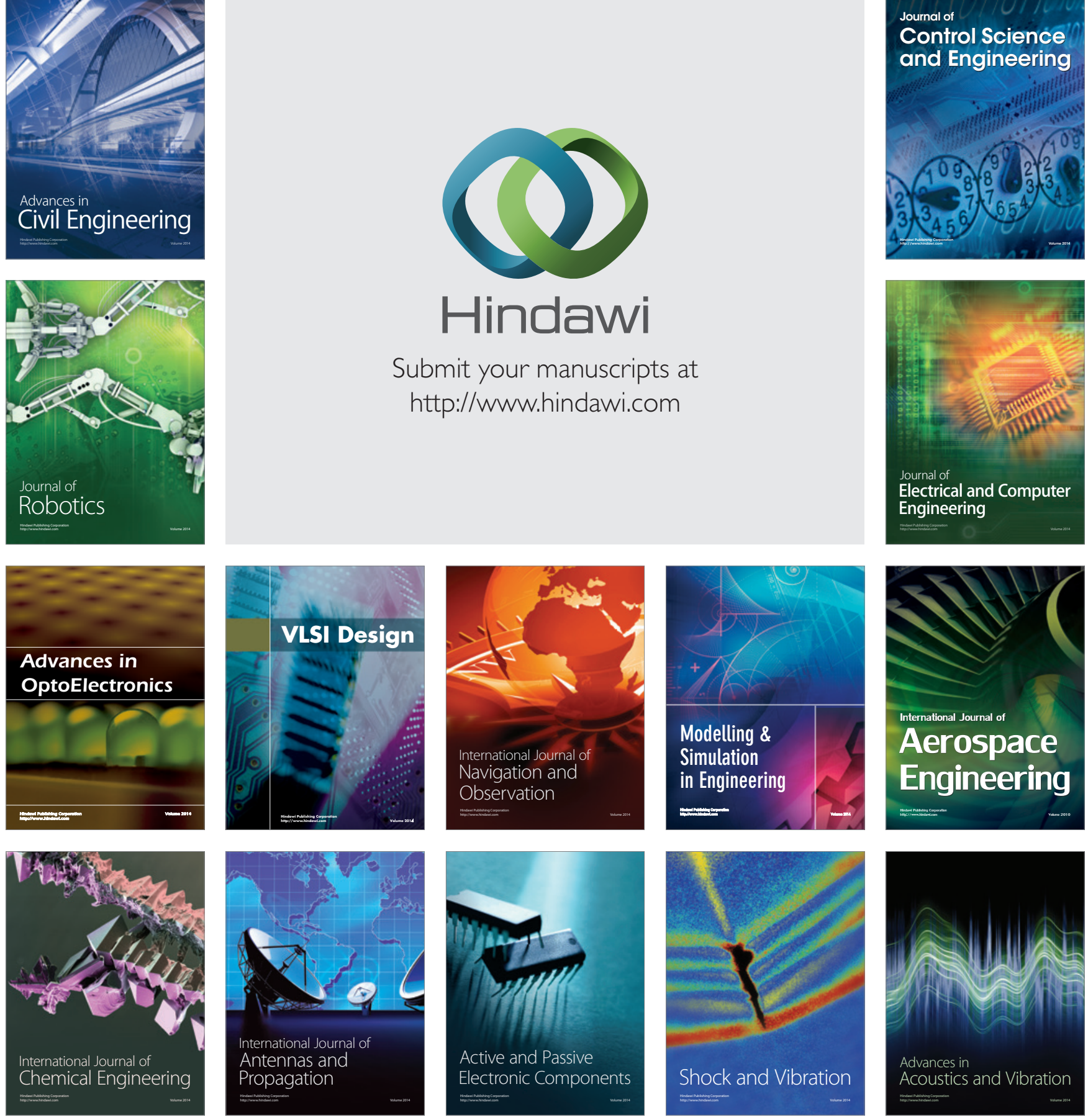